\title{
The Time-of-Flight Detector for the ALICE experiment
}

\author{
M.C.S. Williams for the ALICE collaboration \\ EP Division, CERN, 1211 Geneva 23, Switzerland
}

\begin{abstract}
The Multigap Resistive Plate Chamber (MRPC) will be used to build a large Timeof-Flight detector for the ALICE experiment. It will cover an area of $150 \mathrm{~m}^{2}$ consisting of 160,000 channels of $3.5 \times 2.5 \mathrm{~cm}^{2}$ read-out pads. We present the results of the last 2 years of $R \& D$ during which we investigated problems associated with scaling up from single cells of $3 \times 3 \mathrm{~cm}^{2}$ to strips with active area of $7 \times 120 \mathrm{~cm}^{2}$ read out with 96 pads.
\end{abstract}

Key words: resistive plate chambers, ALICE, time of flight, particle identification

\section{Introduction}

Particle identification for the ALICE experiment is a key ingredient of the ALICE detector. To identify particles in the momentum range 0.5 to $2 \mathrm{GeV} / \mathrm{c}$ a time-of-flight array is needed. Events of high multiplicity are expected for $\mathrm{Pb}$ $\mathrm{Pb}$ collisions (up to 8,000 particles per unit of rapidity); thus the time-of-flight detector has to be highly segmented. The present design is $\sim 150 \mathrm{~m}^{2}$ in area and consists of 160,000 read-out channels each with an area of $2.5 \times 3.5 \mathrm{~cm}^{2}$. Two years ago [1] the multigap resistive plate chamber was selected as the detector of choice for the ALICE Time-of-Flight detector. This decision was based on results from small prototype chambers. Even though it is conceivable to build the ALICE array from 160,000 individual read-out cells, it is much easier to build larger detector units that have a segmented readout. The Time-of-Flight array proposed [2] for ALICE consists of detector elements that are long strips with an active area of $7 \times 120 \mathrm{~cm}^{2}$. These strips have 96 read-out pads, each with an area of $3.5 \times 2.5 \mathrm{~cm}^{2}$. These are arranged in two rows of 48 pads. The principal goal of the R\&D effort during the last year was to investigate 
the problems associated with scaling up from a single cell $\left(3 \times 3 \mathrm{~cm}^{2}\right.$ active area) to an ALICE TOF strip.

\section{Description of Multigap Resistive Plate Chamber}

The MRPC was developed 6 years ago [3]. It consists of a stack of resistive plates with electrodes connected to the outer surfaces. A cross section of a strip designed for the ALICE experiment is shown in figure 1. Glass is used as the resistive plate material. Spacers separate these plates and thus form gas gaps; the size of these gaps is $250 \mu \mathrm{m}$. Many strips have been constructed; typically the internal glass plates have a thickness of $550 \mu \mathrm{m}$, while the outer glass plates are $2 \mathrm{~mm}$ thick. The voltage is applied to the outer surfaces using a layer of carbon tape with a resistivity of $2 \times 10^{5} \Omega$ /square. These voltage layers are insulated from the read-out pads with $350 \mu \mathrm{m}$ thick layer of Mylar; thus both anode and cathode read-out pads can be at ground potential. The cathode signal is brought down to the lower board by the connector shown in figure 1 . Since there is a connector for each pad, there are 48 such connectors on each side of the strip; thus they also aid the mechanical stability of the strip by fixing the distance between the two PCBs. Nylon fibres (fishing line) were used to define the size of the gas gap; these spacer fibres ran across the width of the strip at the boundaries between pads (every $2.5 \mathrm{~cm}$ ). The strip had a length of $1.22 \mathrm{~m}$; the length of the active area was $1.2 \mathrm{~m}$. The high voltage to the carbon tape layer was attached to the $1 \mathrm{~cm}$ space at each end of the strip. The voltage could be applied either as a high negative voltage to the cathode carbon layer, with the anode carbon layer at ground potential, or as a positive and negative high voltage to the anode and cathode carbon layers respectively. The edges of the internal glass plates defined the edge of the active area. We examined the data to see if there was an excess of large signals (streamers) associated with particles passing close to these edges; we did not detect any sign of such behaviour.

\section{Results}

The front-end electronics used for all the measurements shown here consisted of a commercial amplifier, MAXIM 3760, which is a very fast $(560 \mathrm{MHz})$ transimpedance preamplifier, followed by a high speed comparator (AD 96685). Since we are using fixed threshold discriminator, the time measurement has a dependence on the pulse height due to the finite rise time of the signal; all measurements presented here are after this correction (we term this the $\mathrm{T}(\mathrm{A})$ correction). In figure 2.a we show a typical time distribution obtained from 
testing one read-out pad of a 96 pad strip. The tails, outside the Gaussian fit, are small; at this level (a) they have no impact on the physics program of the ALICE experiment and (b) we are not sure if they originate in the detector or are produced in the measuring system itself. In figure 2.b we show a histogram of time resolution and efficiency measured for 17 pads randomly distributed along the length of the strip. In this case the detector was constructed with 5 gaps of $230 \mu \mathrm{m}$ each. The data presented in figure 2.b was taken at a voltage of $12.5 \mathrm{kV}$. These results are very similar to those obtained with a single cell [4]. An obvious question is : what gap tolerance is required? To investigate this we constructed 3 strips where the only difference was the size of the gas gap. The sizes were 220, 250 and $280 \mu \mathrm{m}$ and each strip was constructed with 6 gas gaps. In figure 3 we show the efficiency and the time resolution of the three strips. It is clear that at $16.5 \mathrm{kV}$ all three strips are on the plateau for the efficiency and are close to the best time resolution. We also show the change in absolute time versus applied voltage. The absolute time shown is after $\mathrm{T}(\mathrm{A})$ correction, where the coefficients are calculated from the $18 \mathrm{kV}$ data.

\section{Discussion}

We find that changing the size of the gas gap by $\pm 30 \mu \mathrm{m}$ results in very little change in operating voltage. Thus it is not too surprising that strips of $1.2 \mathrm{~m}$ in length have a very uniform response. A reason for this insensitivity could be as follows: at a given applied voltage a device with a smaller gas gap will have a higher electric field, which will result in an increase in the Townsend coefficient; thus the gain will be higher. However the avalanche grows in size with the distance it travels; thus a smaller gap results in a smaller distance and

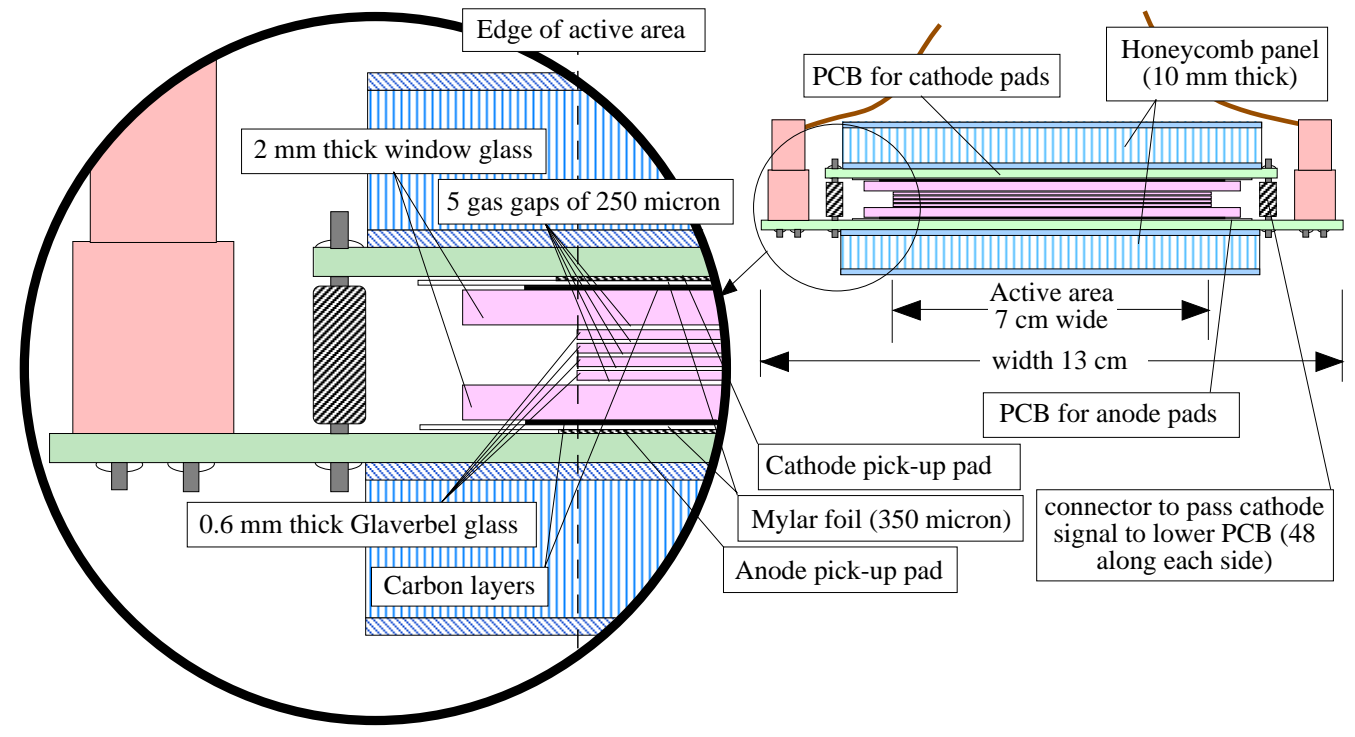

Fig. 1. Cross section of MRPC strip with 96 pads for the readout. 


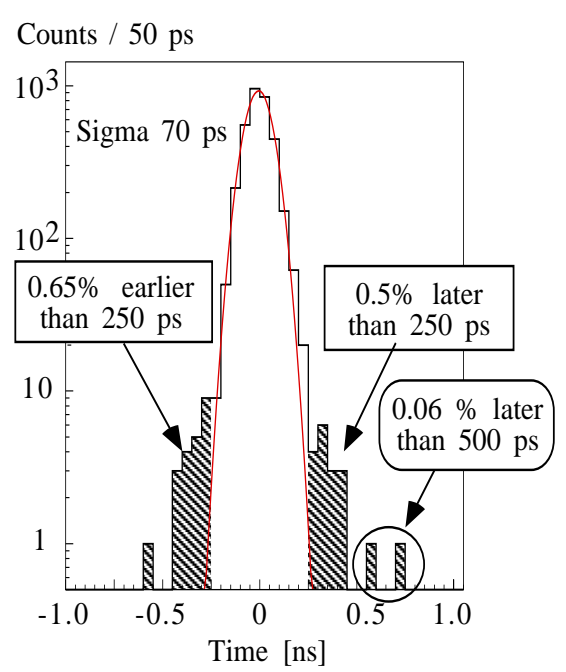

$a$
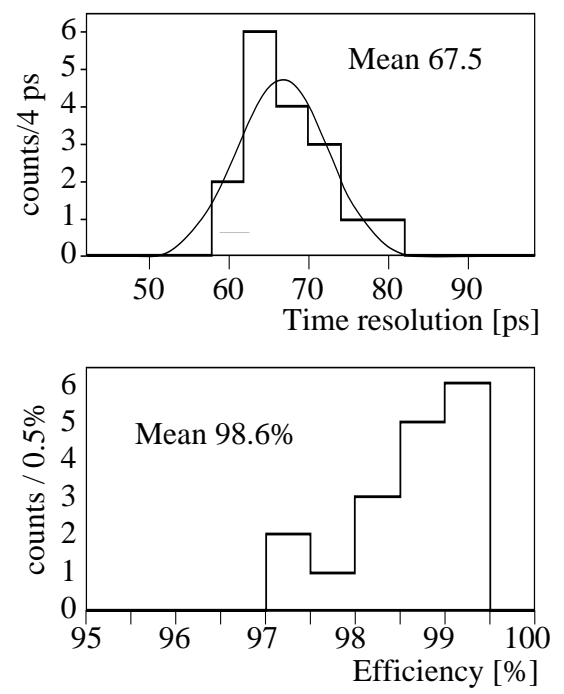

$b$

Fig. 2. a. T(A) corrected time distribution; b. Time resolution and efficiency distributions.
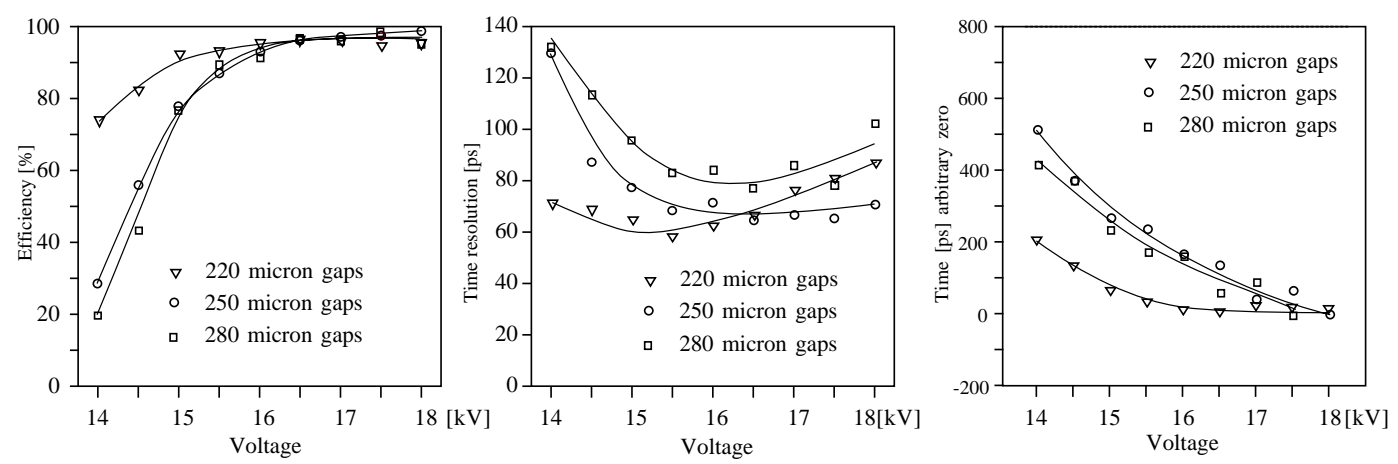

Fig. 3. Efficiency, time resolution and time walk as a function of the high voltage.

thus the gain is lower. It seems that we are working in a regime where these effects cancel. A similar insensitivity to gap size has been observed with the Micromegas chamber [5]. The very small shift in absolute time with voltage (especially with the $220 \mu \mathrm{m}$ chamber) gives us confidence that the timing of this device will not be overly sensitive to changes in high voltage, pressure and temperature. Further studies are in progress. We have identified a commercial amplifier that has a very good performance with the chamber. This amplifier is now the base-line front-end for the ALICE TOF detector.

\section{References}

[1] M.C.S. Williams et al., Nucl. Phys. A, Vol 661 (1999)707.

[2] ALICE: Technical Design Report of the Time of Flight System (TOF), 
CERN/LHCC 2000-12, ALICE TDR 8, 16 February 2000.

[3] E. Cerron Zeballos, I. Crotty, D. Hatzifotiadou, J. Lamas Valverde, S. Neupane, M.C.S. Williams and A. Zichichi, Nucl. Instr. Meth. A374(1996)132.

[4] A. Akindinov et al., Nucl. Instr. Meth. A456(2000)16.

[5] Y. Giomataris, Nucl. Instr. Meth. A419(1998)239. 